\title{
Un produit emblématique à la dérive des continents et des consommateurs : I'huile d'olive
}

\author{
Giulia PALMA ${ }^{1}$ \\ Martine PADILLA ${ }^{1}$ \\ Institut agronomique méditerranéen, \\ 3191 route de Mende \\ 34093 Montpellier cedex 5, \\ France \\ <palma@iamm.fr > \\ Article reçu le 13 juillet 2012 \\ Accepté le 30 juillet 2012
}

Le marché de l'huile et corps gras est un marché de forte compétition. Largement incorporé dans les produits industriels, souvent mis en cause par les Plans nationaux de santé en raison des graisses saturées jugées peu favorables à la santé, le marché des corps gras est dominé par I'huile de soja asiatique ou brésilienne (33\%) et par I'huile de palme (18\%). Bien qu'elle ne représente que $3 \%$ des huiles végétales produites au monde (Mili, 2006), I'huile d'olive bénéficie, elle, d'un crédit de confiance grâce à ses propriétés bienfaitrices. Elle occupe une place très importante dans toutes les cultures méditerranéennes et, dans les dernières décennies, elle gagne du terrain au niveau de la planète. Comment l'évolution des échanges commerciaux internationaux a-t-elle eu des répercussions en termes de consommation auprès des populations des pays traditionnellement producteurs de cette huile, à savoir les pays méditerranéens ? Comment se situe aujourd'hui I'huile d'olive chez le consommateur du monde ? Telles sont les questions majeures de cet article.

Apres avoir tracé un état des lieux mondial de la production d'huile

\begin{abstract}
Drift of an emblematic mediterranean product: the case of olive oil Mediterranean countries, in particular Spain, Italy and Tunisia, rule the international market of olive oil, in terms of land, production and exports. However, several new countries, such as Argentina, Chile, the United States and Australia have entered the market and are becoming important actors: their production, as well as imports of olive oil, are growing fast. Thanks to the positive image that it acquired due to its health benefits, olive oil is more and more appreciated in countries where its consumption is recent and non traditional. On the contrary, in Mediterranean countries, namely in Tunisia, other vegetable oils and fats tend to substitute olive oil, which is more expensive. However, such oils and fats could be responsible for the increase of cardiovascular diseases and obesity.
\end{abstract}

Key words: olive oil, consumption, trade, Mediterranean

d'olive, on s'intéressera aux exportations et importations ainsi qu'aux disponibilités au niveau du consommateur au sein des pays méditerranéens et dans quelques pays non méditerranéens témoins. Nous nous intéresserons ensuite à la place désormais occupée par I'huile d'olive par rapport aux autres huiles végétales. À partir du cas de la Tunisie, les liens en termes de prévalence de l'obésité seront également soulignés.

\section{La Méditerranée, une zone productrice d'huile d'olive toujours omniprésente}

L'olivier étant une plante emblématique de la Méditerranée, il n'est pas étonnant que la plupart de la superficie mondiale dédiée à cette culture se trouve, justement, dans le Bassin méditerranéen. $C^{\prime}$ est ici que se concentrent $95 \%$ de la production et $85 \%$ de la consommation mondiale (Mili, 2006).

La moitié des oliviers restent des productions de type traditionnelles, qui assurent $40 \%$ de la production, alors que les restants $60 \%$ se repartissent entre une production intensive (30\% des hectares et $50 \%$ de la production) et une production marginale (20\% de la surface et $10 \%$ de la production) (Mili, 2006).

L'Espagne occupe une position d'absolue prédominance : en 2010, les oliviersy occupaient plus de 2 millions d'hectares, à savoir $22 \%$ de la surface mondiale dédiée à cette culture. Viennent ensuite la Tunisie (18\%) et I'Italie (13\%), avec respectivement 1,6 million et 1,2 million d'hectares. En Grèce et en Turquie, les surfaces cultivées sont pratiquement équivalentes (environ 800000 hectares) et représentent de l'ordre $9 \%$ de la surface mondiale, suivies du Maroc avec 735000 hectares (8\% des surfaces mondiales). Enfin, en Syrie, 647500 hectares (7\%) sont occupés par des oliviers (FAOSTAT).

De manière prévisible, à une grande superficie cultivée en oliviers correspond une production élevée en huile d'olive. Encore une fois, l'Espagne est de loin le plus grand producteur d'huile d'olive au monde, avec presque 1,5 million de tonnes produites en 2010, assurant à elle seule $45 \%$ de la production mondiale (figure 1) (FAOSTAT). En deuxième

Pour citer cet article : Palma G, Padilla M. Un produit emblématique à la dérive des continents et des consommateurs : I'huile d'olive. OCL 2012 ; $19(5)$ : 283-289. doi : $10.1684 /$ ocl.2012.0467 


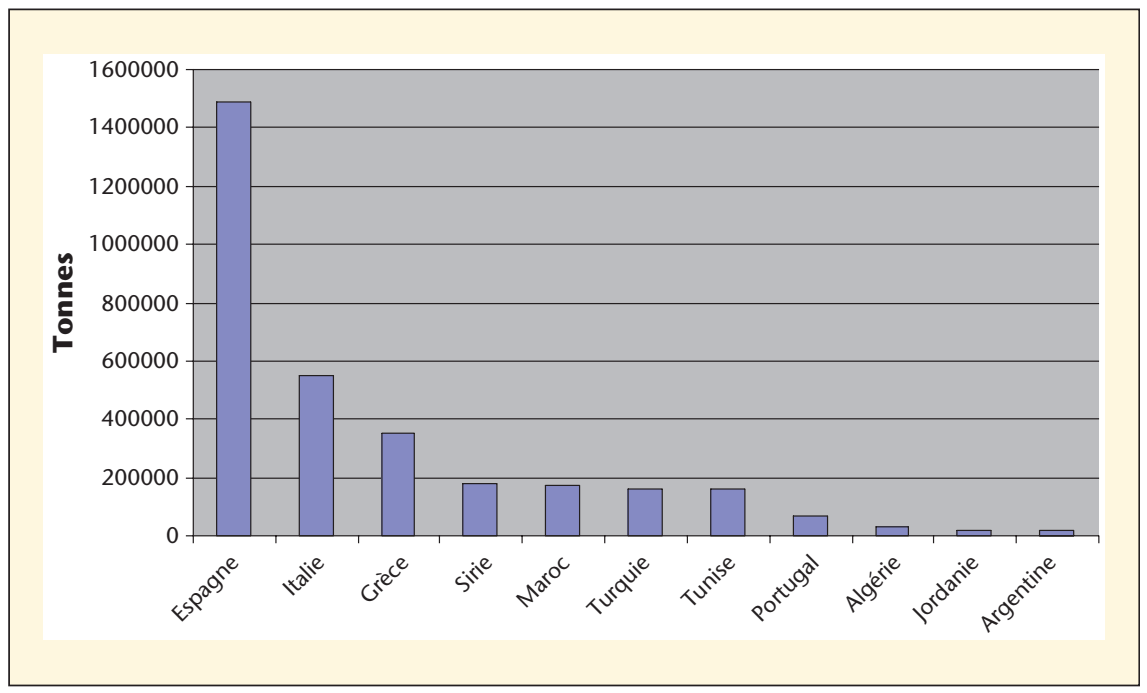

Figure 1. Production d'huile d'olive en tonnes (année 2010).

Source des données: FAOSTAT.

position, mais avec un écart important, se place l'Italie avec un peu plus que 548500 tonnes (17\% de la production mondiale), suivie par la Grèce avec 352800 tonnes (11\%). La Syrie, bien que $7^{\mathrm{e}}$ pays par la superficie cultivée en oliviers est le $4^{\mathrm{e}}$ pour la production d'huile d'olive (117 400 tonnes représentant $5 \%$ de la production totale). Les autres pays qui ont une production élevée (environ $5 \%$ de la production mondiale) sont le Maroc, la Turquie et la Tunisie. Cette dernière produit également une quantité d'olives de table considérable, ce qui explique l'écart entre surface plantée en oliviers et production d'huile d'olive (FAOSTAT).

\section{Des concurrents qui progressent. . .}

Si l'on s'intéresse à l'évolution de la production sur les cinquante dernières années (1961-2010), on observe que la production mondiale a globalement augmenté de $20 \%$. Toutefois, une analyse plus approfondie montre des différentiels d'évolution importants selon les décennies. En effet, entre 1961 et 1971, la production d'huile d'olive a baissé considérablement, à savoir de $42 \%$. Jugée non rentable, concurrencée par des huiles plus productives, on a alors cru cette production condamnée à terme. Cette tendance négative a continué jusqu'en 1981, mais avec une chute beaucoup moins Source des données: FAOSTAT. importante (- $8 \%$ ). C'était compter orce de la science qui, maladies cardiovasculaires (Lég des lobbies très puissants, notamment aux États-Unis. À partir de là, la production a constamment augmenté, notamment entre les années 1981 et 1991, où elle a progressé de $59 \%$. Tous les concernés par des chutes et des progressions irrégulières tout au long de cette période, mais le cas de l'Espagne est particulièrement intéressant car ce pays a plus que doublé sa production entre 1991 et 2001. En effet, grâce à une relance des plantations et à une utilisation plus rationnelle des techniques de culture, la production a fortement augmenté, notamment en 2001/ 2002 et 2003/2004 qui se caractérisent par des campagnes de récoltes extraordinaires (IOC).

L'engouement international pour cet " or liquide» a bouleversé les sites de production. Bien qu'originaire du bassin méditerranéen, la culture de l'olivier s'est répandue dans plusieurs pays du monde, notamment là où le climat était particulièrement adapté à cette production : I'Argentine, l'Australie, le Chili et les États-Unis, qui sont devenus respectivement les $10^{\mathrm{e}}, 18^{\mathrm{e}}, 20^{\mathrm{e}}$ et $21^{\mathrm{e}}$ producteurs du monde. En Australie par exemple, qui compte aujourd'hui 30300 hectares d'olivier et plus de 6000 tonnes d'huile d'olive, la culture était introduite avec les premiers colonisateurs, mais jusqu'aux années 1950 elle ne s'est pas développée (figure 2). En effet, le produit était peu connu et la demande faible, les coûts de production élevés et les huileries industrielles étaient rares. L'arrivée des émigrants en provenance des pays méditerranéens a stimulé le marché intérieur qui aujourd'hui est en nette expansion (IOC). De même, la demande en huile d'olive des émigrés latins en Argentine a joué un rôle clé : on y trouve aujourd'hui 55700 hectares d'oliviers et

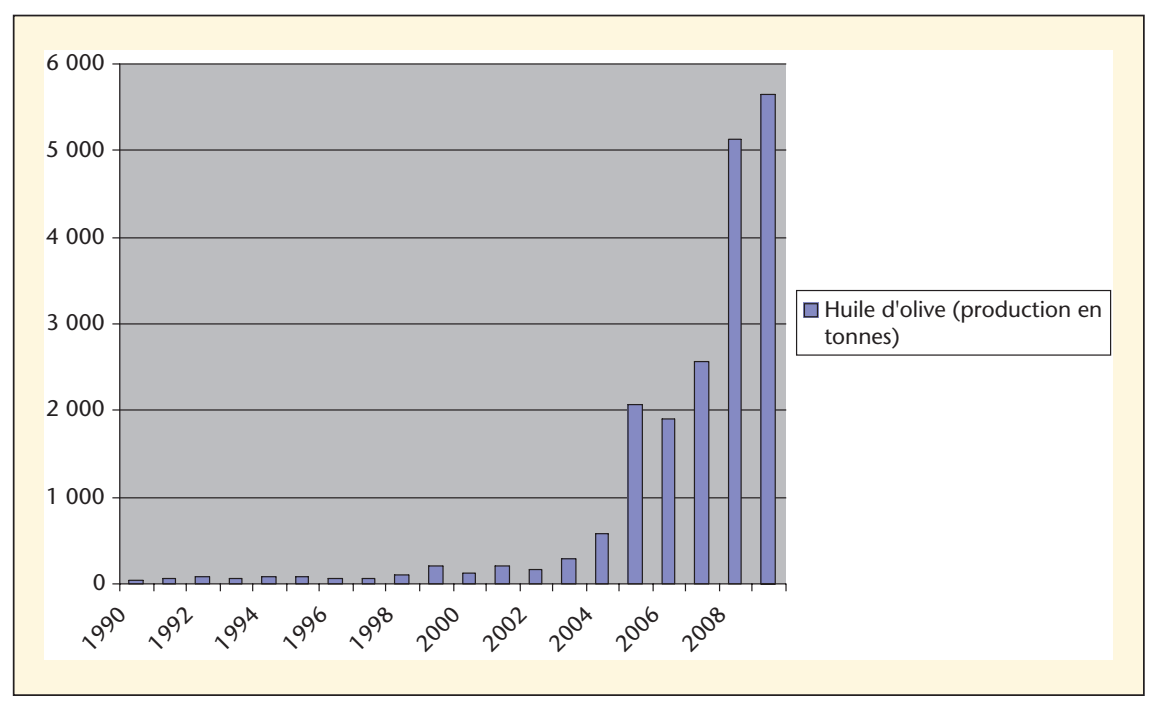

Figure 2. Production d'huile d'olive en Australie (en tonnes).

(1) 
une production de plus que 16000 tonnes d'huile d'olive. Évidemment, le Bassin méditerranéen reste la zone de production par excellence, en concentrant la majeure partie de l'huile d'olive produite, mais l'analyse des évolutions de mises en production en dehors de cette zone, montre une augmentation très significative. Entre 1990 et 2009, en Argentine et au Chili, la production d'huile d'olive a plus que doublé. L'augmentation est encore plus frappante en Australie, où en seulement 9 ans (2000-2009) la production a été multipliée par 45 et a atteint près de 6000 tonnes, ce qui est très significatif si on considère que jusqu'aux années 1990 elle était pratiquement inexistante. De manière similaire, la production américaine, notamment californienne, a vu son volume multiplié par 4 entre 1990 et 2009 (FAOSTAT).

Au-delà des mouvements migratoires qui expliquent la mise en production de I'olivier, I'expansion du marché de I'huile d'olive est aussi le résultat de plusieurs facteurs. À partir des études de Ancel Keys (Keys, 1975) qui ont souligné un lien direct entre alimentation, taux de cholestérol et morbidité due aux maladies cardiovasculaires, un grand nombre d'études ont démontré les bienfaits en termes de santé de ce qu'on appelle " la diète méditerranéenne " ${ }^{1}$. Au-delà des propriétés nutritionnelles des fruits et légumes, du poisson et des légumineuses qui abondent dans ce régime, plusieurs de ses bienfaits sont attribuables notamment à I'huile d'olive. II est reconnu aujourd'hui que cette dernière participe à la réduction du taux du "mauvais cholestérol ", protège par conséquent des maladies cardiovasculaires et possède des propriétés antioxydantes (Léger, 2000 ; Descomps, 2000). Depuis, dans plusieurs pays, comme aux EtatsUnis, au Royaume-Uni et en Australie, les recommandations nationales en termes de nutrition s'inspirent du modèle méditerranéen. Grâce aussi aux médias qui ont permis une vulgarisation et divulgation de ces nouvelles connaissan-

\footnotetext{
${ }^{1}$ Diète méditerranéenne : régime caractérisé par une consommation élevée de graines, fruits et légumes, légumineuses, poisson et huile d'olive, et au contraire une faible consommation de viande rouge et de graisses animales. Un style de vie actif et la convivialité des repas participent aussi à la définir.
}

ces, la diète méditerranéenne, et avec elle I'huile d'olive, profitent d'une image très positive surtout dans des pays qui ne sont pas des consommateurs traditionnels de cet aliment. La redécouverte de la " bonne cuisine " et du plaisir de cuisiner, ainsi que la recherche de santé dans I'alimentation, ont amené à un engouement pour la cuisine méditerranéenne dans son ensemble (cuisine italienne, espagnole, grecque, libanaise, etc.) qui demande une utilisation presque exclusive d'huile d'olive comme matière grasse. Enfin, la grande distribution a favorisé sa diffusion et a permis de garder un certain équilibre sur le marché international. En effet, selon certains auteurs (Mili, 2006), la recherche de nouveaux débouchés pour le marché de l'huile d'olive dans des pays nouveaux consommateurs à partir des années 1990 a répondu à la nécessité de placer une production qui dépassait la demande dans les pays méditerranéens.

Cet ensemble hétérogène de facteurs a donc fait que la demande d'huile d'olive a énormément augmenté hors Méditerranée, ce qui a entraîné, d'une part, un accroissement des importations de ces biens en provenance des zones de production traditionnelles, mais en même temps, étant un marché rémunérateur, une mise en production ou un élargissement des plantations en dehors de la Méditerranée. Pour se rendre compte de l'importance de la diffusion de I'huile d'olive, il suffit de remarquer qu'en 2000 elle était présente dans presque un tiers de ménages nord-américains, et environ $40 \%$ des ménages canadiens et anglais, et que ces chiffres ont augmenté rapidement : seuls $6,7 \%$ des ménages américains utilisaient I'huile d'olive en 1987, ils étaient très minoritaires en Angleterre vingt ans plus tôt (Mili, 2006).

\section{Une forte concentration des exportations pour un marché élargi}

Plus des trois quarts des exportations mondiales d'huile d'olive ne concernent que trois pays : I'Espagne (49\%), I'Italie (22\%) et la Tunisie (10\%) (figure 3). L'Espagne, avec 660694 tonnes exportées en 2009, confirme encore une fois son rôle clé du marché. En 2007, $53 \%$ de l'huile produite en Espagne a été exportée (FAOSTAT), notamment vers I'Italie (FAOSTAT). Les autres pays destinataires, mais beaucoup moins importants, sont la France, I'Australie, les Etats-Unis et le Royaume Uni. La plus grande partie de l'huile exportée est de qualité élevé : $59 \%$ d'huile extravierge et $20 \%$ d'huile vierge (IOC).

Trois pays nouveaux producteurs $d^{\prime}$ 'huile d'olive gagnent de l'importance dans les exportations : c'est le cas de l'Argentine, de l'Australie et des États-Unis, qui sont respectivement les $7^{\mathrm{e}}, 10^{\mathrm{e}}$ et $11^{\mathrm{e}}$ exportateurs mondiaux.

Bien qu'exportatrice d'huile d'olive, I'Italie en est en même temps le premier importateur: avec 467076 tonnes importées en 2009, elle assure un tiers

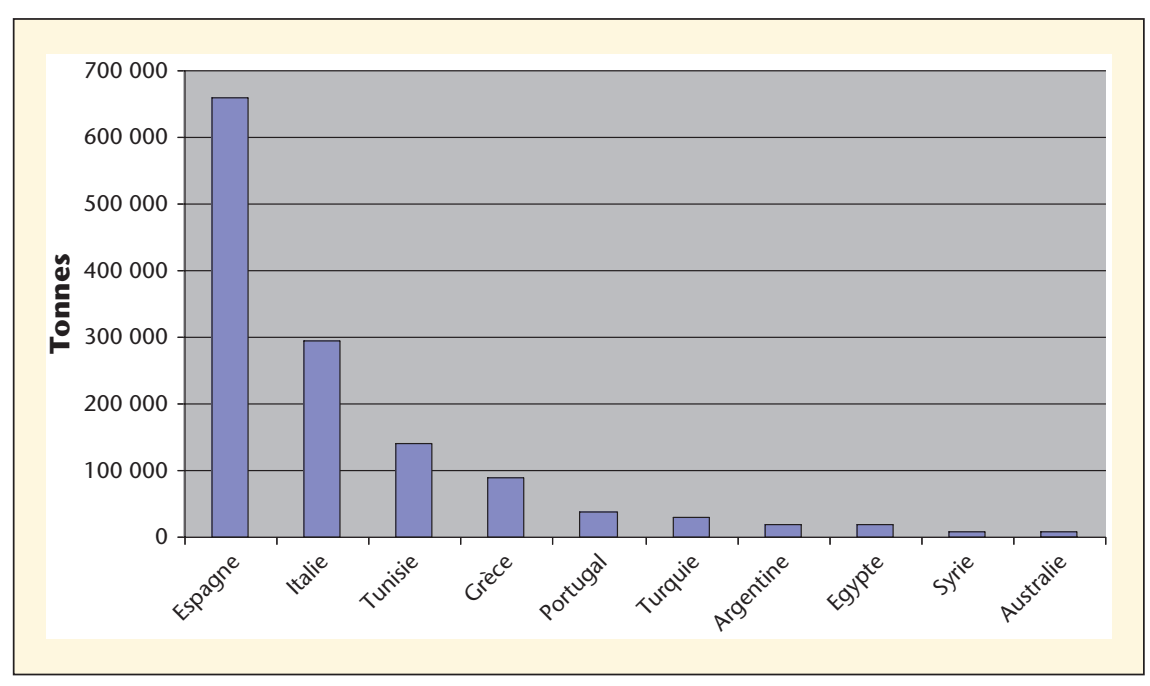

Figure 3. Exportations d'huile d'olive en tonnes (année 2009) des principaux pays producteurs. Source des données : FAOSTAT. 
des importations mondiales. Suivent les États-Unis (18\% des importations totales) et la France (8\%) (figure 4 ). Nous pouvons faire I'hypothèse que l'Italie est une plaque tournante : elle achète I'huile des autres pays (souvent en vrac) pour le revendre ensuite à des pays tiers, en bénéficiant au passage de la valeur ajoutée. En effet, I'Italie importe massivement de l'Espagne, ainsi que de la Tunisie et de la Grèce. L'analyse des flux $d^{\prime}$ exportation indique que I'huile est ensuite exportée de I'Italie avant tout vers les États-Unis, et d'autres pays au monde comme le Canada, la France, la Chine, I'Espagne, I'Australie et le Royaume Uni. De son côté, la Tunisie exporte tout d'abord en Italie, ensuite aux États-Unis, en France, en Espagne et au Maroc.

D’autre pays méditerranéens producteurs importent également des quantités non négligeables d'huile d'olive, et comme évoqué précédemment, parmi les pays non-méditerranéens nous retrouvons le Royaume-Uni, I'Allemagne, le Brésil, le Japon, le Canada, I'Australie. Cette dernière importe de plus en plus $d^{\prime}$ 'huile d'olive en provenance de l'Union européenne (Italie, Espagne), jusqu'à 43000 tonnes par an, en multipliant les quantités par 4 depuis 1990. Nous observons la même tendance en Afrique du Sud (+700 \% des importations entre 1990 et 2008), au Japon (+ $700 \%$ ), et aux États-Unis (+ $152 \%)$.

En Chine, le phénomène est tout aussi important : les importations atteignent en 2008 un niveau 162 fois plus important qu'en 1990. Bien que I'huile de soja et de palme restent largement les plus consommées en Chine, I'huile d'olive est de plus en plus demandée, notamment dans le milieu urbain aisé. Les ventes ont augmenté à un rythme plus que soutenu entre 2004 et 2008, avec une hausse moyenne annuelle de $+35 \%$ (Lazzeri, 2011). La production est encore très limitée, et donc le marché reste un marché d'importation. L'Espagne, I'Italie et la Grèce sont les principaux fournisseurs avec, respectivement, $40 \%, 30 \%$ et $20 \%$ des importations (IOC). Viennent ensuite la Turquie, la Tunisie et la France. Toutefois, d'autres acteurs se placent sur ce nouveau marché prometteur: c'est le cas de l'Australie, qui a trouvé dans la Chine un bon acheteur de son huile de qualité haut de gamme. Pour avoir une idée de la progression de I'intérêt du géant asiatique pour I'huile d'olive, il suffit de s'attarder sur les importations qui ont progressé fortement, ainsi que sur le fait qu'un salon dédié à I'huile d'olive se tient chaque année depuis 2004 à Shanghai (Lazzeri, 2011).

\section{L'attractivité du marché international prive le consommateur méditerranéen de son huile d'olive}

En termes de consommation, les pays grands consommateurs d'huile d'olive

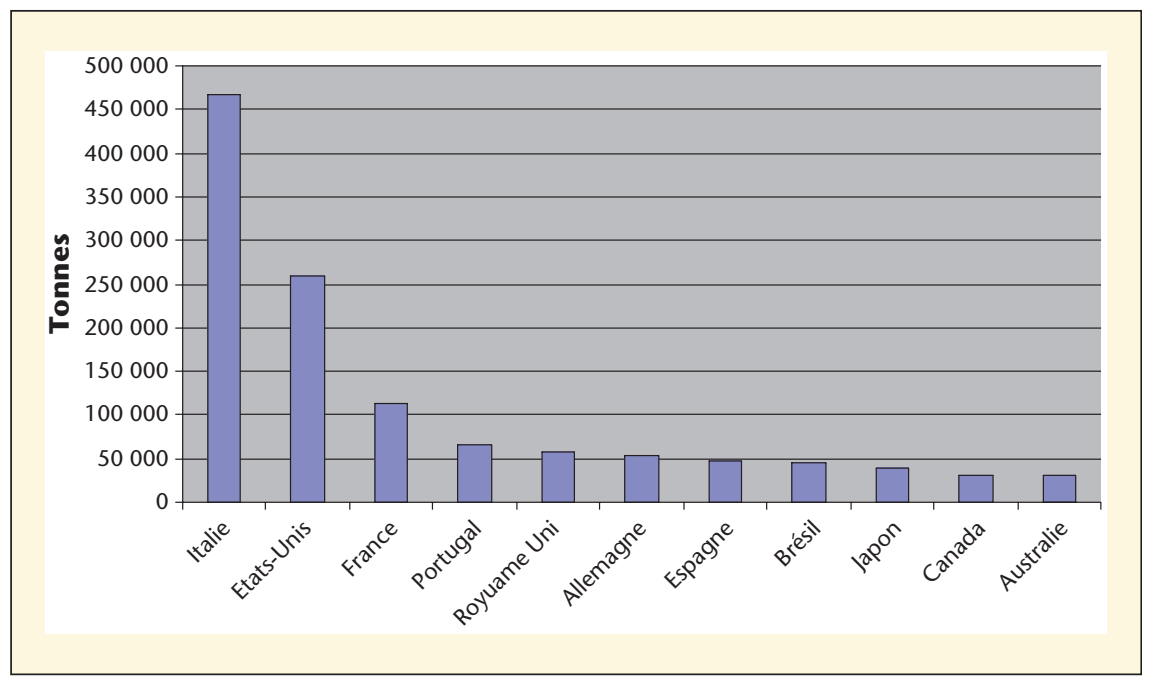

Figure 4. Importations d'huile d'olive en tonnes (année 2009) des principaux pays importateurs.

Source des données : FAOSTAT. sont bien évidemment les pays méditerranéens (tableau 1). Toutefois, des changements importants sont en cours dans plusieurs pays du bassin qui semblent s'éloigner du "régime traditionnel ", caractérisé entre autres par une consommation élevée d'huile d'olive. En même temps, certains pays hors zone se rapprochent de plus en plus de la diète méditerranéenne (Palma, 2012 ; Padilla, 2012). Néanmoins, la cuisine des pays du Bassin reste basée sur I'huile d'olive et un grand nombre de plats l'incorporent comme ingrédient fondamental. Plusieurs techniques de conservation des aliments se basent également sur l'usage d'huile d'olive. Cette huile est aussi largement utilisée en cosmétique, en tant que médicament, mais aussi lors des cérémonies religieuses et trouve son utilisation aussi dans des rites de traditions populaires (contre le mauvais œil, etc.). Elle a aussi une valeur symbolique très importante, car fortement liée au terroir, à la tradition, provenant d'un arbre séculaire, qui aménage une grande partie du territoire méditerranéen en le caractérisant de façon unique.

De loin les plus grands consommateurs d'huile d'olive, la Grèce et I'Italie, avec respectivement $16 \mathrm{~kg}$ et $14 \mathrm{~kg}$ par habitant par an, se placent en premières positions. Viennent ensuite l'Espagne (12 kg/hab/an) et le Portugal (5 kg/hab/ an). Dans les autres pays méditerranéens, les niveaux sont beaucoup plus faibles, alors même qu'ils affichent des quantités produites importants. C'est le cas par exemple de la Tunisie (3 kg/hab/an), du Maroc (3 kg/hab/ an), de l'Algérie $(0,7 \mathrm{~kg} / \mathrm{hab} / \mathrm{an})$ et, cas frappant : la Turquie $(0,7 \mathrm{~kg} / \mathrm{hab} / \mathrm{an})$. Cette dernière est le $7^{\mathrm{e}}$ producteur $\mathrm{d}^{\prime}$ huile d'olive au monde, mais la consommation y est plus faible qu'en Australie, Canada, Royaume Uni ou encore Suède ! De plus, en Turquie, la part de I'huile d'olive dans le total des huiles végétales utilisées ne représente que 3,5\%. La consommation d'huile d'olive reste concentrée dans la zone Sud et Est du pays, alors que la margarine (à base de graisses végétales) est de plus en plus consommée dans le pays entier (FAO, 2005). II est évident que certains pays grands producteurs, comme la Turquie et la Tunisie, favorisent I'exportation de I'huile d'olive au détriment de la consommation domestique. En Turquie, I'exportation a plus que triplé entre 1990 et 2004, alors 
Tableau 1. Classement des pays majeurs par consiommation d'huile d'olive (en kg/habitant/an), par consommation d'huile d'olive/ huiles totales (\%) et par huile d'olive/matieres grasses totales (\%).

\begin{tabular}{|c|c|c|}
\hline $\begin{array}{l}\text { Pays par consommation } \\
\text { d'huile d'olive (kg/habitant/an) }\end{array}$ & $\begin{array}{l}\text { Pays par consommation d'huile } \\
\text { d'olive/huiles totales }(\%)\end{array}$ & $\begin{array}{l}\text { Pays par huile d'olive/matières } \\
\text { grasses totales }(\%)\end{array}$ \\
\hline Grèce $(15,9)$ & Grèce $(60,5)$ & Grèce $(28,7)$ \\
\hline Italie (14) & Italie $(50,7)$ & Italie $(24,2)$ \\
\hline Espagne $(11,8)$ & Espagne $(42,5)$ & Espagne $(21,1)$ \\
\hline Portugal $(4,9)$ & Portugal $(27,6)$ & Maroc $(12,3)$ \\
\hline Tunisie $(3,4)$ & Maroc $(24,7)$ & Tunisie $(10,7)$ \\
\hline Maroc $(2,9)$ & Tunisie $(19,4)$ & Portugal $(9,5)$ \\
\hline Australie $(2,1)$ & Malte $(13,4)$ & Australie (4) \\
\hline France $(1,7)$ & Australie $(10,7)$ & France $(2,8)$ \\
\hline Liban $(1,1)$ & France $(8,4)$ & Algérie $(2,7)$ \\
\hline Malte (1) & Albanie $(8,2)$ & Liban $(2,7)$ \\
\hline Canada $(1,1)$ & Liban $(6,1)$ & Malte $(2,4)$ \\
\hline Royaume Uni $(0,9)$ & Suède $(5,1)$ & Canada $(2,1)$ \\
\hline États-Unis $(0,9)$ & Algérie (5) & Turquie $(1,8)$ \\
\hline Suède $(0,8)$ & Royaume Uni $(4,7)$ & Suède $(1,8)$ \\
\hline Turquie $(0,7)$ & Canada $(4,5)$ & Albanie $(1,8)$ \\
\hline Algérie $(0,7)$ & Turquie $(3,5)$ & Royaume Uni $(1,7)$ \\
\hline Albanie $(0,6)$ & États-Unis $(3,2)$ & États-Unis $(1,5)$ \\
\hline Japon $(0,2)$ & Égypte $(2,4)$ & Japon $(0,6)$ \\
\hline Chili $(0,2)$ & Chili $(1,8)$ & Chili $(0,6)$ \\
\hline Égypte $(0,1)$ & Japon $(1,4)$ & Égypte $(0,5)$ \\
\hline Afrique du Sud $(0,1)$ & Afrique du sud $(0,9)$ & Afrique du sud $(0,3)$ \\
\hline Argentine $(0,1)$ & Argentine $(0,6)$ & Argentine $(0,2)$ \\
\hline
\end{tabular}

même que la production a augmenté de seulement $57 \%$ (IOC).

Jadis matière grasse exclusive dans les pays méditerranéens, l'huile d'olive devient souvent marginale dans les usages. Si elle joue un rôle encore important en Grèce (60\% de toutes les huiles), un peu moins en Italie (51\%), en Espagne (42\%), au Portugal (28\%) et au Maroc ( $25 \%)$, dans les autres pays méditerranéens, elle ne représente plus que 2 à $19 \%$ de toutes les huiles consommées. En France, à Malte, au Maroc, en Italie et en Espagne, la consommation d'huile d'olive a augmenté de manière significative entre 1960 et 2007 (figure 5). En revanche dans certains pays du Bassin méditerranéen, la consommation par habitant a chuté grandement : elle a diminué de $80 \%$ en Turquie, $59 \%$ au Liban, $45 \%$ en Albanie et Tunisie, $23 \%$ au Portugal. À titre d'exemple, en 1960, $78 \%$ des huiles consommées par habitant en Tunisie étaient de I'huile d'olive, alors qu'au- jourd'hui elle ne représente que $19 \%$. Même phénomène en Albanie où l'huile consommée était pour $56 \%$ de I'huile d'olive en 1960 pour tomber à $8 \%$ en 2007. Des situations similaires se présentent en Turquie, au Portugal et au Liban. Dans ces pays, les huiles qui se sont substituées à l'huile d'olive traditionnelle sont surtout le tournesol en Albanie (83\% des huiles végétales hors huile d'olive) et au Portugal (44\%), tournesol et huile de palme en Turquie (30 et $24 \%$ respectivement), alors qu'en Tunisie et au Liban, c'est le soja (74 \% et $47 \%$

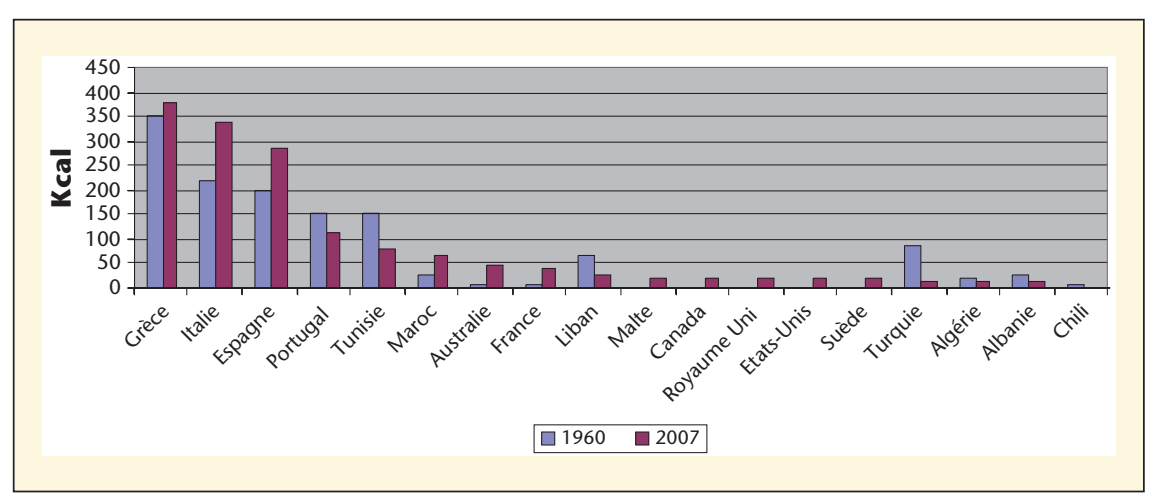

Figure 5. Évolution de la consommation d'huile d'olive entre 1960 et 2007 (en kcal). Source des données : FAOSTAT. 
respectivement). Parmi d'autres facteurs, les prix nettement inférieurs des huiles de graines par rapport à I'huile d'olive, favorisent sans doute leur adoption au sein des ménages méditerranéens. La Tunisie représente un cas exemplaire, qui mérite d'être analysé avec plus de détail.

\section{La substitution de I'huile d'olive par des huiles mélangées nuit à la santé. Le cas de la Tunisie}

Bien que $7^{\mathrm{e}}$ producteur mondial et $3^{\mathrm{e}}$ exportateur d'huile d'olive au monde, la consommation en Tunisie de cette huile est devenue faible. De $78 \%$ des huiles consommées dans le pays il y a 50 ans, elle ne représente aujourd'hui que $19 \%$ du total. En effet, I'huile d'olive était traditionnellement la principale matière grasse, mais la situation a changé grandement. À partir de 1962, le gouvernement tunisien a mené des actions pour favoriser l'exportation de I'huile d'olive à l'étranger et garantir à la population locale une consommation $\mathrm{d}$ 'huile moins chère. Pour cela les huiles de graines importées, ou des mélanges d'huiles, ont été subventionnées et leur prix stabilisé. En 2003, la consommation des huiles de graines était passée à $17 \mathrm{~kg}$ par habitant par an, alors que dans les années 1970, elle était de $6 \mathrm{~kg}$ (Ali, 2003; Bechir, 2003). II s'agit surtout d'huile de soja et de colza, qui bénéficient $d^{\prime}$ un soutien à la consommation grâce au paiement d'une partie des coûts d'importation par l'État. Cela a créé une disparité très importante entre les prix à la consommation de I'huile d'olive et ceux des huiles de graines, le rapport étant tout en faveur de ces derniers. En effet, si en 1971-75 I'huile d'olive valait deux fois plus chère que l'huile de graines, en 1996-2000 le rapport était de 1 à 4 (Ali, 2003 ; Bechir, 2003).

Un autre facteur qui semble avoir favorisé le changement est le fait que I'habitude de cuisiner en friture, rend les autres huiles végétales plus appropriées que I'huile d'olive (FAO, 2005). Nous observons donc que la consommation des huiles de graines ne cesse d'augmenter au détriment de celle de I'huile d'olive. La consommation de cette dernière est sensible au prix (demande élastique) et les deux produits sont considérés comme substituables, alors qu'il y a une grande différence de qualité nutritionnelle. II y a de plus en plus d'évidence que si certaines huiles, notamment d'olive, de colza et de tournesol présentent des bénéfices pour la santé, d'autres, comme I'huile de palme et de coprah (coco), largement utilisées dans l'industrie agroalimentaire, sont accusées de favoriser la survenance des maladies cardiovasculaires. Pour cela, de nombreux pays, comme la Suisse, I'Autriche, et le Danemark, ont interdit les acides gras trans, et la Finlande et la Roumanie envisagent des taxes. De plus, depuis octobre 2011, le Danemark a introduit une taxe sur les produits alimentaires riches en tout type de graisses saturées. Cela met donc en danger les graisses concurrentes de l'huile d'olive sur le marché international et peut engendrer à terme un renouveau du marché de I'huile d'olive.

En 2002, les huiles végétales (huile d'olive incluse) représentaient $16 \%$ de la DEA (disponibilité énergétique alimentaire) des habitants de la Tunisie. L'évolution entre 1965 et 2002 ne semble pas montrer de changements significatifs de ce point de vue. En effet, l'apport énergétique total de la diète tunisienne a augmenté tout au long des années et la proportion dédiée aux huiles est restée stable. Toutefois, si on regarde la quantité effective d'huile (toutes huiles inclues) consommée en Tunisie, on s'aperçoit qu'elle est passée de $42 \mathrm{~g} /$ personne/jour en 1965 à $59 \mathrm{~g} /$ personne/jour en 2000 ! Nous pouvons déduire que cette augmentation a concerné les huiles végétales en dehors de I'huile d'olive. En effet, la consommation de cette dernière montre une diminution très importante $(-45 \%)$ entre 1960 et 2007 (FAOSTAT) alors que les kcal provenant des huiles végétales hors huile $d^{\prime}$ 'olive ont vu leur volume multiplié par 8 .

$Y$ aurait-il une relation avec l'évolution de l'obésité dans ce pays ? Entre 1980 et 2000, l'obésité a doublé chez les hommes et femmes (FAO, 2005). La consommation accrue de matières grasses végétales est probablement, avec d'autres facteurs comme l'excès de sucre et la sédentarité, à la base de l'accroissement de surpoids et obésité dans le pays. Selon une enquête menée en 2007 portant sur 8006 Tunisiennes adultes (35-70 ans), $16 \%$ des hommes et $38 \%$ des femmes étaient obèses (Tahina, 2006).

\section{Conclusion}

Si la Méditerranée reste encore la zone de production par excellence de I'huile d'olive, de nouveaux concurrents rentrent sur ce marché prometteur. Au niveau des consommateurs, le prix non compétitif de l'huile d'olive par rapport aux autres huiles végétales, induit une baisse de sa consommation dans les pays du bassin méditerranéen, alors que dans le passé elle était la matière grasse presque exclusive. En revanche, les pays nouveaux consommateurs en dehors de cette zone, montrent un intérêt de plus en plus croissant pour I'huile d'olive, notamment grâce à la "découverte " des bienfaits pour la santé qui lui sont attribués. En Méditerranée, la substitution entre l'huile d'olive et les mélanges d'autres huiles végétales, et en parallèle I'augmentation de la consommation de graisses dans leur ensemble, dont la Tunisie montre un cas d'école, sont en revanche associées à l'aggravation des taux de surpoids et d'obésité des populations.

Ainsi, il semblerait que le consommateur méditerranéen soit dépourvu de I'huile d'olive qui est en revanche destinée au marché d'exportation. Le risque est aussi que les pays traditionnellement producteurs perdent leur position hégémonique dans le marché international, et soient sérieusement concurrencés par les "nouveaux " producteurs. Demain, la Méditerranée serat-elle consommatrice d'huile d'olive produite " ailleurs »?

\section{Conflits d'intérêts : aucun}

\section{RÉFÉRENCES}

Ali C, Bechir SM. La demande de I'huile d'olive en Tunisie : une analyse économétrique. New Medit 2003 ; 2 : 26-8.

FAO. Profil Nutritionnel de la Tunisie. Rome : 2005.

FAOSTAT http://faostat.fao.org/.

IOC http://www.internationaloliveoil.org/.

Keys A, Keys M. How to Eat Well and Stay Well. The Mediterranean Way. New York: Doubleday, 1975. 
Lazzeri Y. Chine, un marché émergent pour I'oléiculture. Lettre de veille du CIHEAM 2011 ; $16: 8-10$.

Léger CL, Descomps B. Huile d'olive, coproduits de l'huilerie d'olive et olive-fruit : données actuelles et perspectives concernant la relation aliment et santé. In : Besançon, Debosque, Delpeuch et al. (Eds.), Alimenta- tion méditerranéenne et santé : actualités et perspectives. Paris: John Libbey Eurotext, 2000.

Mili S. Olive oil marketing on Non-traditional markets: prospects and strategies. New Medit $2006 ; 5: 27-37$.

Palma G, Padilla $M$. La "méditérraneisation" des modes alimentaires dans le monde. In : CIHEAM, ed. Mediterra 2012 : Ia diète méditerranéenne pour un développement régional durable. Paris: Sciences Po, 2012.

Tahina, final report. Epidemiological Transition and health impact in North Africa. INCO international scientific cooperation projects (1998-2002). 2006. 